\title{
CONCOMITANT USE OF ORAL ANTIPSYCHOTIC DRUGS AMONG PATIENTS TREATED WITH LONG-ACTING ANTIPSYCHOTIC MEDICATIONS IN AN OUTPATIENT PSYCHIATRIC CARE SETTING
}

\author{
R. Valido ${ }^{1}$, F. Grilo. ${ }^{2}$, M. De Barros ${ }^{1}$, F. Caetano ${ }^{1}$, F. Caldas ${ }^{1}$, P. Frias ${ }^{1}$, P. Ferreira1 ${ }^{1}$, Hospital de Magalhães Lemos, \\ Department of Psychiatry, Oporto, Portugal; University of Porto, Faculty of Economics, Oporto, Portugal.
}

\section{Background and aims}

There have been very few descriptive studies of concomitant use of oral and Long-acting injectable (LAI) antipsychotic medications. The aim of this study was to analyze the use of oral antipsychotics among patients receiving LAls at an outpatient psychiatric care setting.

\section{Materials and methods}

A list of all patients treated with LAI medications at the outpatient service at Hospital de Magalhães Lemos, Oporto, Portugal, between January and December 2018, was obtained from center administrative records. Our sample includes 796 individuals treated with LAI medications. The primary outline was to determine if there was any association between the concomitant use of LAI and oral supplementation with an oral antipsychotic drug and a higher number and/or duration of hospitalizations. Data were processed using STATA13.

\section{Results}

\section{Sociodemographic characterization}

Among 796 patients on LAI medications, $72 \%$ were male and $28 \%$ were female. The mean age was 48,21 years $\left(1^{\circ}\right.$ quartile $-38,25$ years; $3^{\circ}$ quartile - 56 years). Nearly $74 \%$ of the patients were single, $19 \%$ married, $5 \%$ divorced and $1 \%$ widowed. The percentage of nonworking patients was about $90 \%$ (75\% were retired and $15 \%$ were unemployed). Regarding education, $64 \%$ attended elementary school or have no schooling, $19 \%$ attended High School and $16 \%$ obtained higher degrees of education.

\section{Psychiatric Diagnosis}

The vast majority of patients on LAl medication have a diagnosis of Schizophrenia, however mental retardation, Schizoaffective Disorder and Bipolar Disorder have some expression on this population.

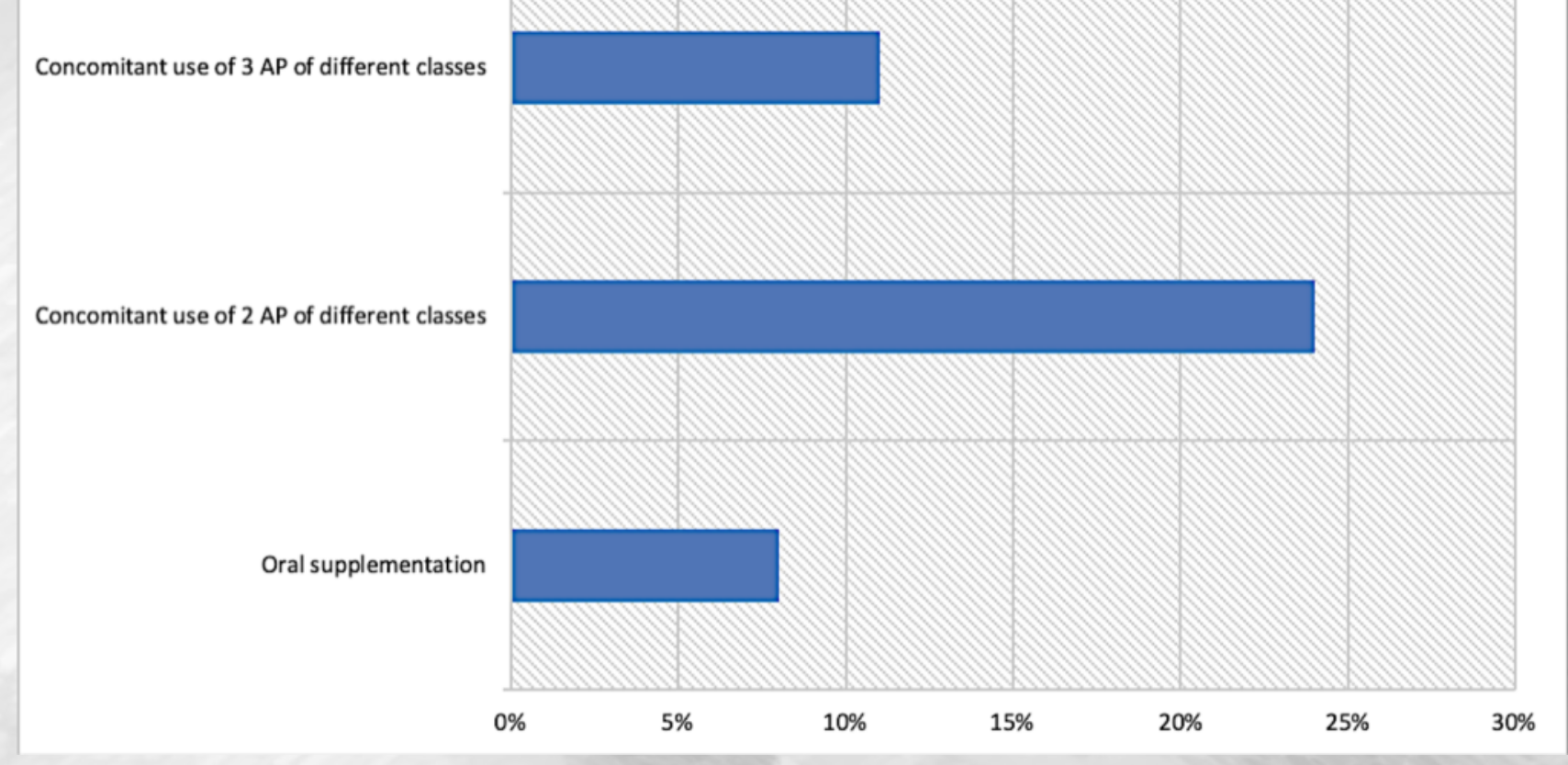

Table 2. Patients under oral supplementation - $11 \%$ (using the oral formulation of the same LAI medication), concomitantly using 2 antipsychotics of different classes - 24\% (1 LAl medication and 1 oral AP) and concomitantly using 3 antipsychotics of different classes $-11 \%$ ( 1 LAl medication and 2 oral AP).

\section{Use of Oral Antipsychotics in association with LAI medication} Roughly $32 \%$ of the patients received at least one oral antipsychotic in association with LAI medication, and about $11 \%$ of patients received treatment with two oral antipsychotics of different classes in association with LAI medication (table 2).

It was found a statistical meaningful association between the duration of the hospitalizations and patients receiving oral antipsychotics and LAI medication, with combined treatment being associated with longer hospitalization times. There was also a significant statistical association between the number of hospitalizations and patients taking oral antipsychotics and LAI medication, being that patients with combined therapy have more hospitalizations (mean number of hospitalizations - 4,73)

\section{Conclusions}

There is a propensity to prescribe an oral formulation of the same medication of the LAI medication despite there is no evidence in the literature to support this decision. Oral supplementation with antipsychotics of different classes was prescribed in a manner consistent with current guidelines recommendations. Around onethird of the patients prescribed LAl antipsychotics receive oral antipsychotics confronting the assumption that LAls are used mainly as monotherapy. Patients receiving oral supplementation of any antipsychotic presumably have a higher burden of psychopathology in relation with more advanced disease states, leading to more frequent hospitalizations and requiring more time to achieve clinical stability. 